\title{
Salivary Gland Extract of Kissing Bug, Triatoma lecticularia, Reduces the Severity of Intestinal Inflammation through the Modulation of the Local IL-6/IL-10 Axis
}

\author{
Helioswilton Sales-Campos $\mathbb{D}^{1,},{ }^{1,2}$ Jonatas da Silva Catarino, ${ }^{1}$ Guilherme Augusto Roza, ${ }^{1}$ \\ Rafael Obata Trevisan, ${ }^{1}$ Luisa Menezes Silva, ${ }^{1}$ Juliana Reis Machado $\left(\mathbb{D},{ }^{1}\right.$ \\ Marcos Vinícius da Silva $\mathbb{D}^{1},{ }^{1}$ Leonardo Euripedes Andrade-Silva, ${ }^{1,3}$ \\ Virmondes Rodrigues-Júnior $\mathbb{D}^{1}{ }^{1}$ and Carlo José Freire de Oliveira $\mathbb{D i D}^{1}$ \\ ${ }^{1}$ Instituto de Ciências Biológicas e Naturais, Universidade Federal do Triângulo Mineiro, Uberaba, MG, Brazil \\ ${ }^{2}$ Instituto de Patologia Tropical e Saúde Pública, Universidade Federal de Goiás, Goiânia, GO, Brazil \\ ${ }^{3}$ Departamento de Patologia Clínica, Universidade Federal do Triângulo Mineiro, Uberaba, MG, Brazil
}

Correspondence should be addressed to Helioswilton Sales-Campos; tonsales@fcfrp.usp.br

Received 6 March 2018; Accepted 3 July 2018; Published 22 July 2018

Academic Editor: Martha Lappas

Copyright (c) 2018 Helioswilton Sales-Campos et al. This is an open access article distributed under the Creative Commons Attribution License, which permits unrestricted use, distribution, and reproduction in any medium, provided the original work is properly cited.

\begin{abstract}
Triatomines are known for their role as vectors of the causative agent of Chagas disease. The occurrence of an arsenal of molecules in their saliva is able to suppress vertebrate immune responses. Thus, it is reasonable to assume that the presence of molecules with therapeutic potential in their saliva is able to constrain inflammation in immune-mediated diseases. Thus, mice were exposed to dextran sulfate sodium (DSS) in drinking water uninterruptedly during 6 consecutive days and treated with T. lecticularia salivary gland extract (SGE) $(3,10$, or $30 \mu \mathrm{g})$ or vehicle (saline) ( $n=6$ /group). At the highest dose $(30 \mu \mathrm{g})$, an improvement in clinical outcome and macroscopic aspects of the intestine were observed. This observation was followed by amelioration in histopathological aspects in the colon especially when the doses of 10 and $30 \mu \mathrm{g}$ were used. Regardless of the concentration used, treatment with $T$. lecticularia SGE significantly reduced the levels of the inflammatory cytokine IL- 6 in the intestine. The production of the anti-inflammatory cytokine IL-10 was positively impacted by the concentrations of 3 and $30 \mu \mathrm{g}$. Our results suggest that the presence of molecules in the T. lecticularia SGE is able to attenuate clinical outcome and colon shortening and improve intestinal architecture besides reducing the production of IL- 6 and inducing a local production of IL-10 in the intestine.
\end{abstract}

\section{Introduction}

Triatomines are known for their role as natural vectors of Trypanosoma cruzi, the causative agent of Chagas disease, which is endemic in 21 Latin American countries and in the southern area of the United States [1]. Furthermore, because of migrations of infected people to nonendemic countries, Chagas disease can also be detected in Europe [2], Canada [3], Asia, and Oceania [4]. So far, 153 species of triatomines were identified and those belonging to the Rhodnius, Triatoma, and Panstrongylus genera are the major members of this group [5-7].
The species Triatoma lecticularia is predominantly observed in the United States and Mexico, being found in domestic and peridomestic areas [8]. This species seems to have a great capability to adapt to distinct environments ranging from rain forests to deserts, valleys, and laboratory conditions $[8,9]$. Experimental data also suggested that $T$. lecticularia is an effective vector of $T$. cruzi to human populations [10]. Further, this species is able to adapt and replace other triatomines in the transmission of Chagas disease [9], besides being one of the most important species with potential to transmit Chagas disease in Texas, United States [11]. In nature, this triatomine is associated with 
terrestrial rodents [8]. Hence, this species shows great capability to transmit the causative agent of Chagas disease, to live and adapt under different environmental conditions, and to parasite distinct vertebrate hosts, including humans. These characteristics may be at least partly attributed to a variety of compounds in their saliva allowing them to properly feed, reproduce, and live.

After millions of years of coevolution with vertebrate hosts, triatomines, like other blood-feeding arthropods, developed in their saliva complex mechanisms to suppress vertebrate immune and hemostatic responses [12-14]. For this reason, studies have been focusing on the pharmacological potential of molecules in the saliva from different hematophagous arthropods to treat inflammatory/autoimmune conditions. Treatment using a salivary extract of Phlebotomus papatasi sandflies improved clinical signs, reduced the disease activity, and modulated dendritic cell (DC) and Th17 cell functions in an experimental model of rheumatoid arthritis [15]. Similarly, sialostatin L, a cysteine protease inhibitor identified in the salivary glands of Ixodes scapularis ticks, was able to reduce the severity of experimental autoimmune encephalomyelitis [16] and to prevent the occurrence of asthma in rodents [17]. In addition, the administration of Aedes aegypti saliva presented a beneficial effect on the clinical outcome of intestinal inflammation induced by dextran sulfate sodium (DSS) [18]. The amelioration of the clinical parameters was followed by an inhibition in the production of IFN- $\gamma$, TNF- $\alpha$, IL- $1 \beta$, and IL-5 together with a reduction in the inflammatory area locally in the intestine [18].

Our group has previously demonstrated the in vitro effect of T. lecticularia salivary gland extract (SGE) on DCs stimulated with LPS [14]. The presence of T. lecticularia SGE in DC cultures reduced the production of TNF- $\alpha$, IL- 6 , and IL-12 besides inducing the production of IL-10 in a concentration-dependent manner with no negative impact on cell viability [14]. However, to the best of our knowledge, the therapeutic role of salivary molecules of triatomines in inflammatory diseases has never been addressed. Thus, the unexplored pharmacological potential of triatomine saliva, especially $T$. lecticularia, prompted us to investigate the therapeutic potential of the saliva of this species in the treatment of experimentally induced intestinal inflammation.

\section{Methods}

2.1. T. lecticularia Salivary Gland Extract (SGE). T. lecticularia males and females were reared in the insect facility at the Institute of Biological and Natural Sciences, Federal University of Triângulo Mineiro. Adults of T. lecticularia were cleaned in $70 \%$ ethanol and water to be dissected in saline, and their salivary glands were transferred to a tube containing cold saline. Every three pairs of glands (representing three insects) were added to $10 \mu \mathrm{l}$ of saline. Subsequently, this material was grinded using sterile needles and centrifuged at $10,000 \mathrm{~g}$ to remove insoluble material. The supernatant (SGE) was removed, and the protein concentration was determined by a NanoDrop 2000 spectrophotometer (Thermo Fisher Scientific, Wilmington, DE,
USA). Aliquots were stored at $-80^{\circ} \mathrm{C}$ until use. All specimens used in this study were classified and identified as previously described [19]. The insects used in this experiment were from F2 generation.

\subsection{Mice, Colitis Induction, and Treatment. Male C57BL/6} mice aged 6-8 weeks were maintained on a $12 / 12 \mathrm{~h}$ light/dark cycle with water and food ad libitum in the animal facility at the Federal University of Triângulo Mineiro (UFTM), Brazil. Mice were divided into groups of 6 animals each as follows: naïve animals (control group), healthy mice without intestinal inflammation treated with the highest concentration of T. lecticularia SGE (control $+30 \mu \mathrm{g})$, mice with intestinal inflammation induced by dextran sulfate sodium (DSS, MP Biomedicals, Illkirch, France; molecular weight: 36,000$50,000)$ treated with sterile saline as a vehicle (DSS + saline), and mice with intestinal inflammation treated with T. lecticularia SGE in three different concentrations (DSS $+3,10$, or $30 \mu \mathrm{g})$. For colitis induction, mice were exposed to $3 \%(w / v)$ DSS uninterruptedly in drinking water during 6 consecutive days. Treatment was performed by intraperitoneal (i.p.) route from the beginning of the exposure to DSS (day 0) until the day before euthanasia (day 5). Thus, each mouse was treated with $T$. lecticularia SGE in $0.1 \mathrm{ml}$ sterile saline or saline only. All experimental procedures were reviewed and approved by the Institutional Animal Care and Use Committee of UFTM (protocol number 371) and performed according to the criteria outlined by the Brazilian Society for Laboratory Animal Science (SBCAL).

2.3. Clinical Assessment. Body weight variation and clinical signs of disease were evaluated daily to obtain a clinical disease score for every mouse. Each clinical sign presented by the mice corresponded to one point, and the sum of points for each mouse outlined a clinical score. Clinical scores were determined as previously described [18].

2.4. Euthanasia and Sample Collection. Mice were euthanized on day 6 , and the colon was removed for further analysis. A picture from each intestine was taken, and the intestinal length was analyzed using ImageJ (National Institutes of Health (NIH), Bethesda, MD, United States). Colon samples were divided into smaller sections that were immediately frozen in liquid nitrogen for quantification of nitric oxide (NO) activity. Some sections were immersed into phosphate-buffered saline (PBS)/10\% formaldehyde for paraffin embedding. For cytokine quantification by enzyme-linked immunosorbent assay (ELISA), samples were collected in PBS containing a protease inhibitor (Complete ${ }^{\circledR}$, Roche Pharmaceuticals, Mannheim, Germany).

2.5. Nitric Oxide (NO). NO production was assessed based on the levels of nitrite accumulation in intestinal homogenates using Griess reaction as previously described [20] with some modifications [21]. The readings were performed at $540 \mathrm{~nm}$ in a 96-well plate reader (Perkin Elmer Cetus, San Jose, CA, USA). The total amount of nitrite in the intestinal samples was calculated based on the absorbance of the serial dilution of sodium nitrite standard curve. Then, results were normalized to the dry weight of each intestinal 
section and expressed as micrograms per milliliter per gram of tissue $(\mu \mathrm{g} / \mathrm{ml} / \mathrm{g})$.

2.6. Cytokine Quantification by ELISA. The levels of IL-10, IFN- $\gamma$, TNF- $\alpha$, IL-10, and IL- 6 were determined in tissue homogenates by ELISA following the manufacturer's instructions (BD Biosciences, San Jose, CA, USA). Results were normalized to the dry weight of each intestinal section and expressed as nanograms per milliliter per gram of tissue (ng/ml/g).

2.7. Histopathological Analysis. To assess the impact of exposure to DSS and treatment using T. lecticularia SGE on intestinal architecture, tissue sections were washed with PBS, fixed in $10 \%$ buffered formalin for $24 \mathrm{~h}$, and then processed for paraffin embedding followed by microtome sectioning. $5 \mu \mathrm{m}$ sections were obtained and stained with hematoxylin and eosin ( $\mathrm{H} \& \mathrm{E})$. The following areas were analyzed to verify disturbances induced by DSS and the impact of treatment using the triatomine saliva: mucosa, submucosa, muscle layers, and serosa. Additionally, intestinal sections were also assessed for the presence of edema, inflammatory infiltrate, and epithelial abnormalities.

Morphometry was performed using Image-Pro Insight (Media Cybernetics). The inflammatory infiltrate was determined based on the damaged area containing inflammatory infiltrate divided by the total area of tissue visualized in the acquired image and expressed as a percentage (\%). A trained pathologist who was blinded to the treatment performed the histopathological analysis.

2.8. Data Analysis and Statistics. Normal distribution and homogeneous variance were tested for all of the variables. When the distribution was considered normal and the variance was homogeneous, parametric tests were used: unpaired Student's $t$-test or one-way ANOVA followed by Tukey's posttest. In cases of non-Gaussian distribution of data, the Mann-Whitney or Kruskal-Wallis tests following nonparametric tests followed by Dunn's test were used. The differences were considered significant when $p<0.05$ (5\%). The results were expressed as mean \pm SD. Statistical analysis was performed using GraphPad Prism 6.0 (La Jolla, CA, USA).

\section{Results}

3.1. T. lecticularia SGE Improves Clinical Outcome in DSSInduced Colitis. To evaluate whether the T. lecticularia salivary molecules would be able to improve clinical signs of colitis, mice were i.p. treated with 3,10 , or $30 \mu \mathrm{g}$ of the preparation described in Methods. These doses were chosen based on previous results from our lab (data not shown). Among the different doses tested, the highest one $(30 \mu \mathrm{g})$ led to the amelioration in clinical outcome and overall clinical score, especially when compared to mice treated with $3 \mu \mathrm{g}$ or saline (Figures 1(a) and 1(c), resp.). The beneficial effect of the highest concentration of T. lecticularia SGE (30 and $10 \mu \mathrm{g}$ ) is highlighted by the reduction in postmortem and accumulated scores when compared to the results observed in mice treated with $3 \mu \mathrm{g}$ or saline (Figures 1(d) and 1(e), resp.). Treatment with triatomine SGE seemed not to impact weight loss (Figure 1(b)). Taken together, our results suggest that $T$. lecticularia SGE is able to improve colitis outcome, reducing the disease severity especially when the highest concentration is used.

3.2. Treatment with T. lecticularia SGE Improves Intestinal Shortening and Histopathological Alterations in Mice Exposed to DSS. As we have observed the positive impact of T. lecticularia SGE on colitis outcome, we addressed how this effect could be related to the amelioration in intestinal architecture and local inflammation. Regardless of the concentration used or the nature of treatment (saliva or vehicle), no alterations were observed for the production of $\mathrm{NO}$ (Figure 2(a)). Interestingly, treatment with the highest dose $(30 \mu \mathrm{g})$ improved intestinal shortening when compared to mice treated with the other concentrations ( 3 or $10 \mu \mathrm{g}$ ) or saline alone (Figures 2(b) and 2(c)).

Next, we aimed to investigate whether the preservation of intestinal length was associated with amelioration in local architecture of the organ. Mice treated with the two highest doses $(10$ and $30 \mu \mathrm{g})$ had discrete lamina propria swelling and mononuclear infiltrate in the mucosal and submucosal layers, especially when the dose of $10 \mu \mathrm{g}$ was used (Table 1). Additionally, treatment at the highest doses (10 and $30 \mu \mathrm{g})$ was able to reduce the severity of the damage (Figures 3(c) and $3(\mathrm{~d})$, resp.) in intestinal crypts (Table 1). On the other hand, mice treated with the lowest concentration $(3 \mu \mathrm{g})$ (Figure 3(b)) or vehicle (saline) (Figure 3(a)) had their crypts severely compromised (Table 1). Altogether, these results suggest a positive impact of T. lecticularia SGE on preventing intestinal shortening besides reducing the severity of histopathological alterations in mice exposed to DSS.

3.3. T. lecticularia SGE Modulates Key Cytokines Related to Colitis Outcome. Next, we aimed to elucidate if the amelioration observed in the clinical outcome and intestinal architecture was related to the modulation of cytokines locally in the intestine. Regardless of the concentration of $T$. lecticularia SGE used, a significant reduction in the production of the colitogenic cytokine IL- 6 was observed in the intestine when compared to mice treated with saline only (Figure 4(c)). Additionally, treatment using 3 or $30 \mu \mathrm{g}$ of SGE augmented the levels of IL-10 in the intestine when compared to mice treated with $10 \mu \mathrm{g}$ or vehicle only (Figure 4(d)). No differences were observed for the production of TNF- $\alpha$ (Figure 4(a)), IFN- $\gamma$ (Figure 4(b)), and IL-27 (Figure 4(e)). These results pointed to the beneficial role of the treatment with $T$. lecticularia saliva in the modulation of key cytokines related to colitis worsening.

\section{Discussion}

Triatomines, such as T. lecticularia, are widely known by their role as vectors of Chagas disease. However, the pharmacological potential of their salivary compounds has never been addressed before. Here, we report for the first time the therapeutic effect of T. lecticularia SGE on the outcome of intestinal inflammation induced by DSS. The amelioration 


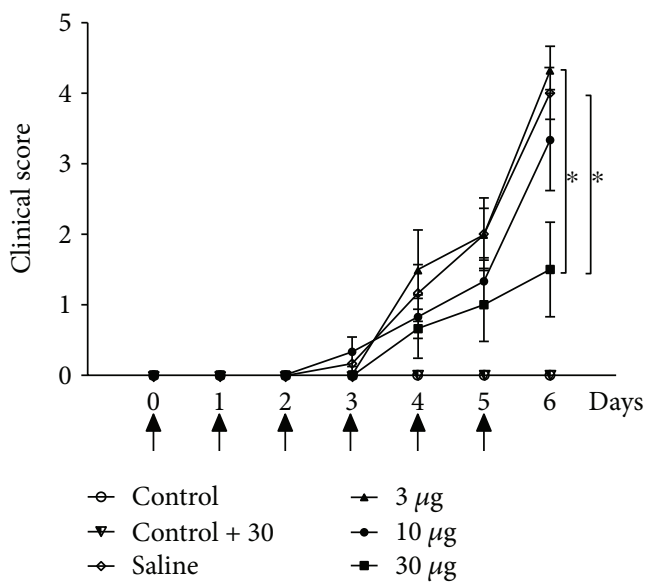

(a)

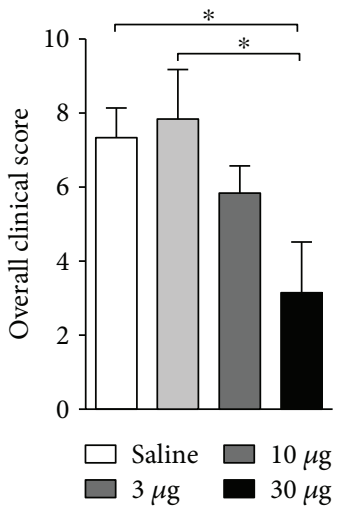

(c)

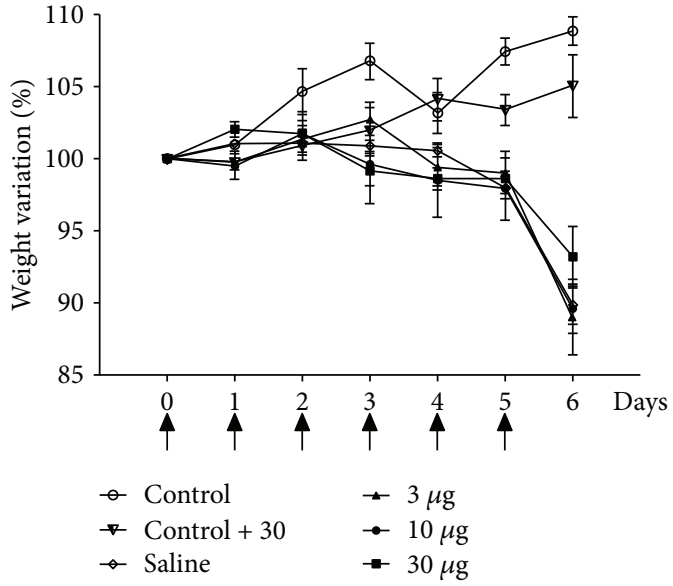

(b)

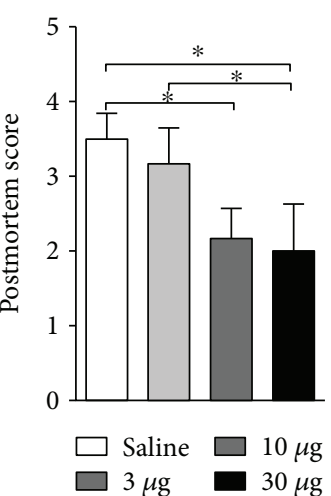

(d)

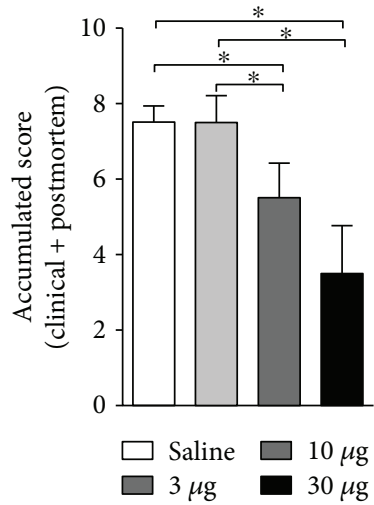

(e)

Figure 1: Treatment with T. lecticularia SGE improves clinical outcome in mice exposed to DSS. Intestinal inflammation was induced by free consumption of drinking water containing 3\% DSS for 6 consecutive days. Mice were treated i.p. with $100 \mu$ l of saline or T. lecticularia $\operatorname{SGE}(3,10$, or $30 \mu \mathrm{g} / \mathrm{animal} /$ day) as depicted in the figure (arrows). The clinical disease score (a), weight variation (b), overall clinical score (c), postmortem score (d), and accumulated score (e) were recorded during the disease development or at the day of euthanasia. Results are representative of two independent experiments ( $n=6$ mice/group). ${ }^{*} p<0.05$.

of clinical and macroscopic aspects seemed to be associated with a reduction in clinical scores, preservation of mucosal architecture, diminishment of local inflammatory infiltrate, and modulation of key cytokines.

The direct correlation between clinical improvement and low rate of side effects represents one of the primary targets in the development of new approaches to treat inflammatory bowel disease (IBD). It is well known that clinical score and its correlated indexes are the main parameters to estimate the level of intestinal inflammation and successfulness of the treatment. However, other aspects, such as colon lengthening, may also be used as an indirect index in this scenario. In the present study, treatment using the highest concentration of $T$. lecticularia saliva besides improving clinical outcome and macroscopic parameters in the intestine also had a positive impact on reducing intestinal shortening.

Immune-mediated diseases, like IBD, are multifactorial disorders where environmental triggers, genetic predisposition, and immune disturbances contribute to disease pathogenesis [22]. In this scenario, current therapies are aimed at modulating the immune system to reduce the severity of inflammation and prolonging periods of remission. Within the immune aspects that dictate the pathogenesis of IBD in the intestinal mucosa, the imbalance between effector and regulatory immune responses plays a central role in disease onset and outcome $[23,24]$. This immune disturbance triggers both innate and adaptive immune responses that in turn produce a wide range of cytokines [25], thus leading to uncontrolled inflammation. For this reason, one of the targets of therapeutic approaches to treat IBD consists in the neutralization of inflammatory players like cytokines. Immunotherapies using neutralizing antibodies targeting TNF- $\alpha$ [26], IL-12/IL-23 subunit p40 [27], or integrins [27] have been successfully used to treat moderate-to-severe Crohn's disease (CD). To note, IBD is especially composed of two different entities CD and ulcerative colitis (UC) [28]. However, a considerable number of patients are refractory or intolerant to these therapies, particularly those targeting TNF- $\alpha$ [29]. This scenario highlights the necessity for developing new therapeutic approaches to treat IBD. In our study, T. lecticularia saliva was effective in the reduction of the colitogenic cytokine IL-6. This molecule is a key modulator in inflammatory response and presents deleterious effects 




(a)

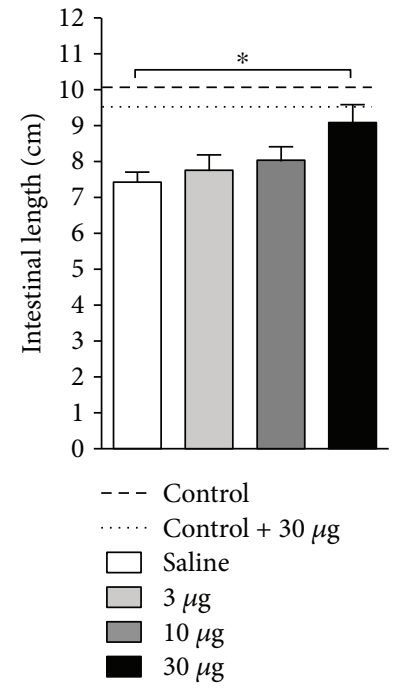

(b)

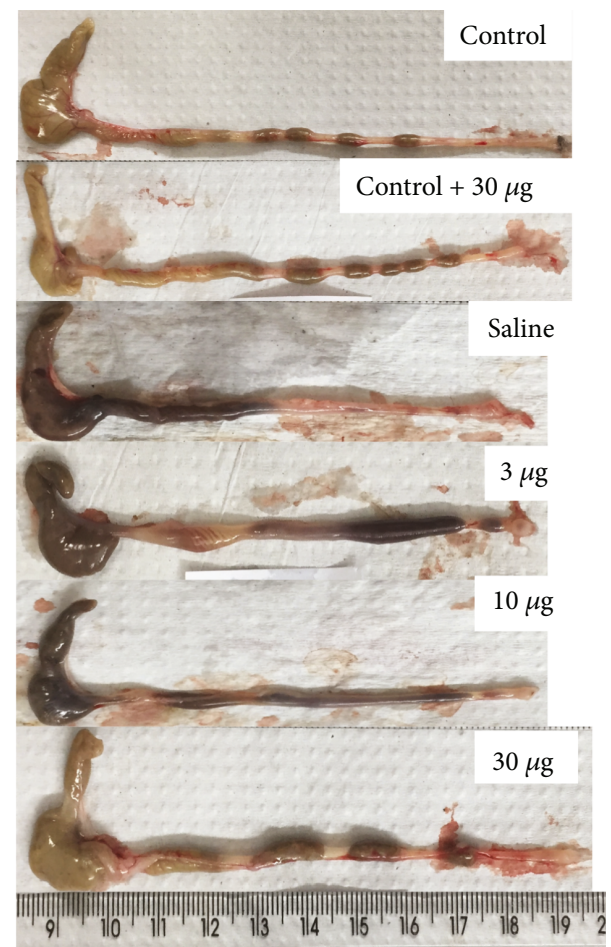

(c)

FIGURE 2: T. lecticularia SGE improves intestinal shortening in mice exposed to DSS. Intestinal inflammation was induced by free consumption of drinking water containing 3\% DSS for 6 consecutive days. Mice were treated i.p. with $100 \mu$ l of saline or T. lecticularia SGE (3, 10, or $30 \mu \mathrm{g} / \mathrm{animal} /$ day). Dashed lines: healthy mice without treatment and not exposed to DSS (control); dotted lines: healthy mice not exposed to DSS and treated with T. lecticularia SGE (30 $\mu \mathrm{g})$. The NO production (a) was assessed as described in Methods. Results were normalized to the dry weight of each intestinal section and expressed as micrograms per milliliter per gram of tissue $(\mu \mathrm{g} / \mathrm{ml} / \mathrm{g})$. The intestinal length $(\mathrm{b}, \mathrm{c})$ was recorded at the day of euthanasia. Results are representative of two independent experiments ( $n=6$ mice/group). ${ }^{*} p<0.05$.

on the pathogenesis of IBD such as inhibition of apoptosis in mucosal $\mathrm{T}$ cells and/or favoring the differentiation of Th17 cells [30]. For this reason, different studies have been targeting IL-6 to treat IBD. A clinical trial conducted with 247 patients induced clinical response and remission, after treatment with anti-IL-6, in refractory patients with moderate-to-severe $\mathrm{CD}$ following failure of anti-TNF therapy [31]. A different clinical trial conducted with a smaller number of individuals $(n=36)$ with active CD also showed the positive effects of treatment targeting IL-6 [32]. Though we have not explored the effects of T. lecticularia saliva on the apoptosis of mucosal $\mathrm{T}$ cells nor its effects on 
TABLE 1: Histopathological alterations in mice exposed to DSS and treated or not with T. lecticularia SGE.

\begin{tabular}{|c|c|c|c|c|c|c|}
\hline Groups & Control & Control $+30 \mu \mathrm{g}$ & Saline & $3 \mu \mathrm{g}$ & $10 \mu \mathrm{g}$ & $30 \mu \mathrm{g}$ \\
\hline Lamina propria swelling & Absent & Absent & Moderate & Moderate & Discrete & Discrete \\
\hline Submucosa swelling & Absent & Discrete & Intense & Moderate & Moderate & Moderate \\
\hline $\begin{array}{l}\text { Mononuclear infiltrate in the } \\
\text { mucosal layer }\end{array}$ & Absent & Absent & Intense & Moderate & Discrete & Moderate \\
\hline $\begin{array}{l}\text { Mononuclear infiltrate in the } \\
\text { submucosal layer }\end{array}$ & Absent & Absent & Moderate & Moderate & Discrete & Moderate \\
\hline Crypts & Preserved & Preserved & Severely damaged & Severely damaged & Dilated & Partly damaged \\
\hline
\end{tabular}



(a)

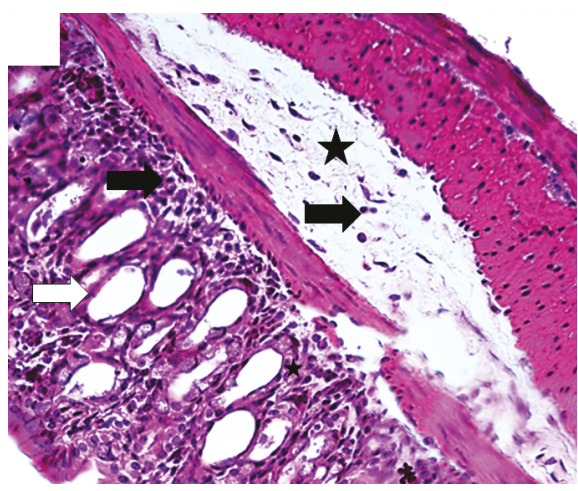

(c)

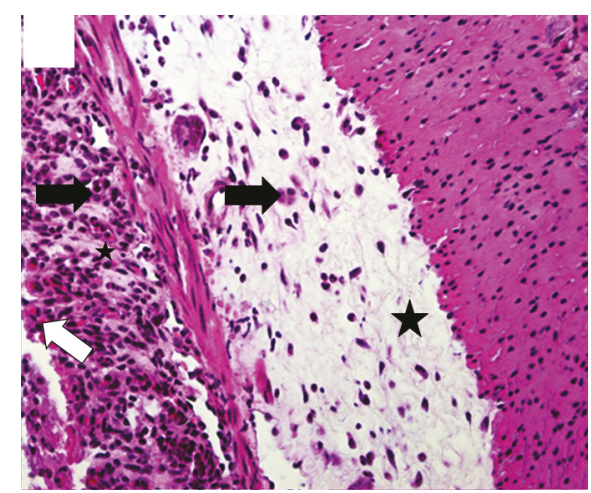

(b)

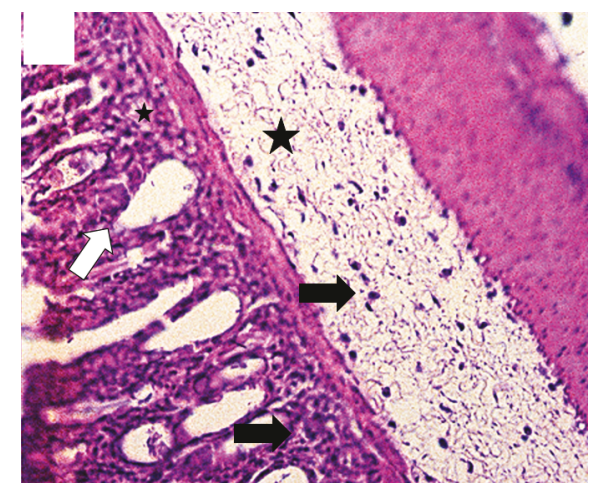

(d)

FIgURE 3: T. lecticularia SGE improves histopathological alterations in mice exposed to DSS. Intestinal inflammation was induced by free consumption of drinking water containing 3\% DSS for 6 consecutive days. Mice were treated i.p. with $100 \mu$ l of saline or T. lecticularia SGE (3, 10, or $30 \mu \mathrm{g} / \mathrm{animal} / \mathrm{day})$. (a) Mice exposed to DSS: lamina propria with moderate edema (asterisk) and intense mononuclear cell infiltration (black arrow) and submucosa with moderate mononuclear infiltrate (black arrow) and intense edema (asterisk). Severely damaged crypts (white arrow); (b) mice exposed to DSS and treated with T. lecticularia SGE ( $3 \mu \mathrm{g} / \mathrm{animal} /$ day): lamina propria with moderate edema (asterisk) and mononuclear cell infiltration (black arrow) and submucosa with moderate mononuclear infiltrate (black arrow) and moderate edema (asterisk). Severely damaged crypts (white arrow); (c) mice exposed to DSS and treated with T. lecticularia SGE (10 $\mu \mathrm{g} / \mathrm{animal} /$ day): lamina propria with discrete edema (asterisk) and mononuclear cell infiltration (black arrow) and submucosa with discrete mononuclear infiltrate (black arrow) and edema (asterisk). Dilated crypts (white arrow); (d) mice exposed to DSS and treated with T. lecticularia SGE (30 $\mu \mathrm{g} /$ animal/day): lamina propria with discrete edema (asterisk) and mononuclear cell infiltration (black arrow) and submucosa with moderate mononuclear infiltrate (black arrow) and edema (asterisk). Partly damaged or dilated crypts (white arrow). Results are representative of two independent experiments ( $n=6$ mice/group).

the differentiation of $\mathrm{T}$ cells, like Th17 lymphocytes, we cannot underestimate its importance in this context. Our group has shown the ability of $T$. lecticularia SGE to reduce the differentiation and production of IL-6 in dendritic cells [14]. Because of the role of these cells in antigen presentation and cell activation or differentiation, the contribution of such modulation to disease amelioration cannot be ruled out.

The reestablishment of immune balance represents a great challenge in the treatment of immune-mediated diseases, like IBD. In our study, treatment with $T$. lecticularia SGE reduced the production of the inflammatory cytokine 


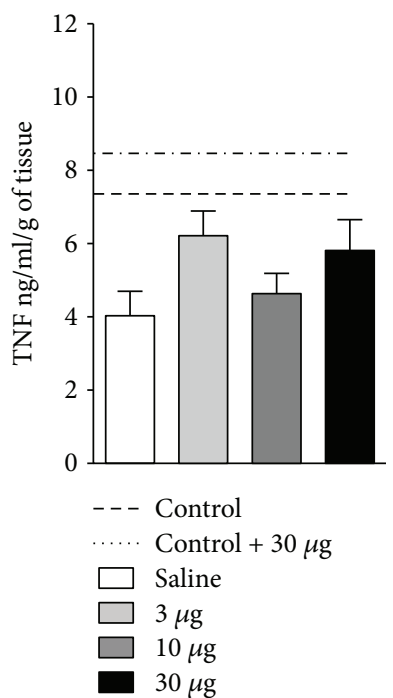

(a)

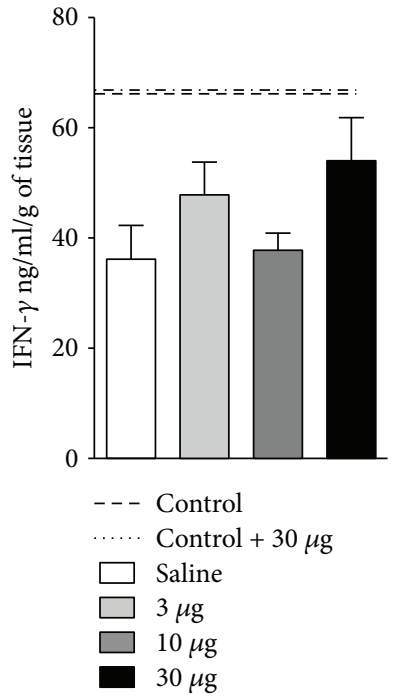

(b)

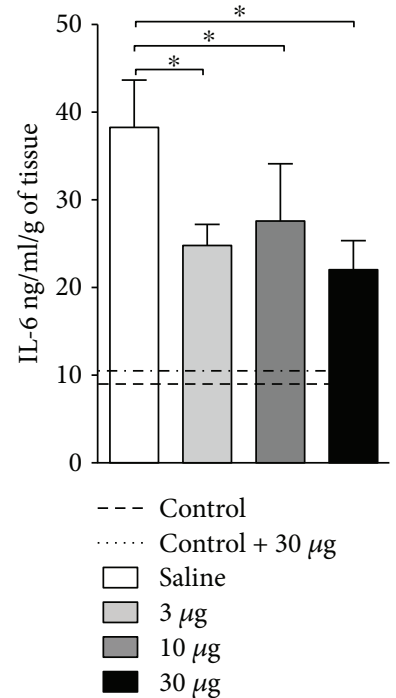

(c)

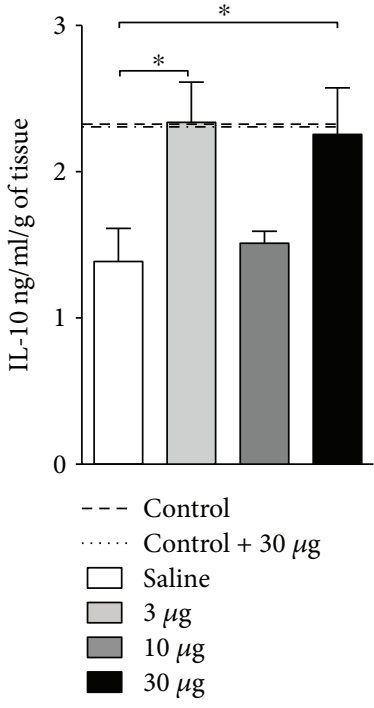

(d)

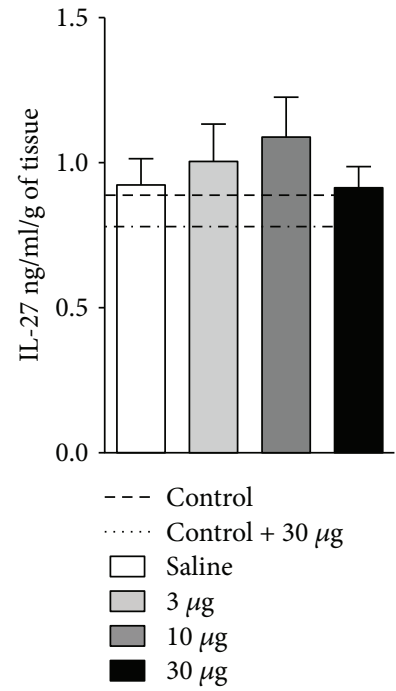

(e)

FIGURE 4: T. lecticularia SGE improved intestinal shortening in mice exposed to DSS. Intestinal inflammation was induced by free consumption of drinking water containing 3\% DSS for 6 consecutive days. Mice were treated i.p. with $100 \mu$ l of saline or T. lecticularia SGE $(3,10$, or $30 \mu \mathrm{g} / \mathrm{animal} /$ day). Dashed lines: healthy mice without treatment and not exposed to DSS (control); dotted lines: healthy mice not exposed to DSS and treated with T. lecticularia SGE (30 $\mu \mathrm{g})$. The concentration of TNF- $\alpha$ (a), IFN- $\gamma$ (b), IL-6 (c), IL-10 (d), and IL-27 (e) was detected in intestinal sections collected with protease inhibitors. Results were normalized to the dry weight of each intestinal section and expressed as nanograms per milliliter per gram of tissue $(\mathrm{ng} / \mathrm{ml} / \mathrm{g})$. Results are representative of two independent experiments ( $n=6$ mice/group). ${ }^{*} p<0.05$.

IL- 6 besides improving the production of IL-10 locally in the intestine. IL-10 presents great anti-inflammatory activity, and mutations in $I L-10$ and its receptor $(I L-10 R)$ were associated with early onset and severity of IBD [33-35]. IL-10 plays a crucial role in the maintenance and regulation of mucosal immune tolerance and in the anti-inflammatory function of macrophages in the intestine [36]. In mice lacking the IL-10R, intestinal inflammation and macrophage dysfunction begin during the third week of life [37]. The role of macrophages in this context is highlighted when these cells are depleted leading to the protection from the development of colitis even in IL-10R-deficient mice [37]. Despite the importance of macrophages in this context, we have only addressed the effects of salivary molecules on the development of DCs. The anti-inflammatory activity of macrophages in the intestinal mucosa seems to be dependent on the IL-10/ p38 $\alpha$ MAPK pathway [38]. The p38 MAPK pathway was formerly involved in the regulation of proinflammatory cytokines by myeloid cells [39]. However, the p38 MAPK cascade seems to have an ambiguous role in inflammation and can present pro- or anti-inflammatory effects [40], depending on the context. Additionally, the production of IL-10 seems also to be negatively influenced by the inhibition of $\mathrm{p} 38 \alpha$, one of the four isoforms of the p38 
MAPK pathway [38]. These results suggest the importance of IL-10 to the maintenance of intestinal homeostasis besides its crucial role in IBD onset and worsening. Furthermore, due to the complexity of targeting one single cytokine to constrain inflammation in IBD, a reduction in IL-6 coupled with higher levels of IL-10 related to the treatment using $T$. lecticularia SGE may represent a promising approach to treat immune-mediated diseases. However, the mechanisms concerning the impact of T. lecticularia SGE on specific aspects of the immune system as well as the molecules responsible for the effects observed still need to be clarified.

\section{Conclusion}

Overall, our data suggested the presence of molecules with pharmacologic potential in T. lecticularia SGE. Treatment with this triatomine SGE besides improving clinical outcome and reducing the severity of damage in the intestinal epithelia also reestablished the immune balance in the intestine. Nonetheless, further studies must be performed in order to elucidate the molecules in T. lecticularia SGE responsible for the effects observed.

\section{Data Availability}

Data are available upon request.

\section{Conflicts of Interest}

The authors declare no conflict of interest.

\section{Acknowledgments}

The authors would like to thank Professor Anderson de Sá Nunes for his intellectual contribution and critical review. The research leading to these results has received support and funding from the Conselho Nacional de Desenvolvimento Científico e Tecnológico (CNPq) (150075/2016-2), Fundação de Amparo à Pesquisa do Estado de Minas Gerais (FAPEMIG), Institutos Nacionais de Ciência e Tecnologia em Entomologia Molecular (INCT-EM) (88887.136392/ 2017-00 - 465678/2014-9), and Coordenação de Aperfeiçoamento de Pessoal de Nível Superior (CAPES).

\section{References}

[1] F. Guhl, A. Auderheide, and J. D. Ramirez, "From ancient to contemporary molecular eco-epidemiology of Chagas disease in the Americas," International Journal for Parasitology, vol. 44, no. 9, pp. 605-612, 2014.

[2] S. Antinori, L. Galimberti, R. Bianco, R. Grande, M. Galli, and M. Corbellino, "Chagas disease in Europe: a review for the internist in the globalized world," European Journal of Internal Medicine, vol. 43, pp. 6-15, 2017.

[3] H. Schipper, B. M. McClarty, K. E. McRuer, R. A. Nash, and C. J. Penney, "Tropical diseases encountered in Canada: 1. Chagas' disease," Canadian Medical Association Journal, vol. 122, no. 2, pp. 165-169, 1980.
[4] J. R. Coura, P. A. Vinas, and A. C. Junqueira, "Ecoepidemiology, short history and control of Chagas disease in the endemic countries and the new challenge for non-endemic countries," Memórias do Instituto Oswaldo Cruz, vol. 109, no. 7, pp. 856-862, 2014.

[5] S. Pita, P. Lorite, J. Nattero et al., "New arrangements on several species subcomplexes of Triatoma genus based on the chromosomal position of ribosomal genes (Hemiptera Triatominae)," Infection, Genetics and Evolution, vol. 43, pp. 225-231, 2016.

[6] C. J. Schofield and C. Galvao, "Classification, evolution, and species groups within the Triatominae," Acta Tropica, vol. 110, no. 2-3, pp. 88-100, 2009.

[7] C. Galvão, Vetores da doença de Chagas no Brasil, Sociedade Brasileira de Zoologia, 2014.

[8] J. Jurberg and J. M. Costa, "The resistance of fasting and nutritional aspects of Triatoma lecticularia (Stal, 1859) (Hemiptera, Reduviidae, Triatominae)," Memórias do Instituto Oswaldo Cruz, vol. 84, no. 3, pp. 393-399, 1989.

[9] Y. Grant-Guillen, B. Nogueda-Torres, J. Gascon-Sanchez, G. Goicochea-Del Rosal, and J. A. Martinez-Ibarra, "Biology of the introduced species Triatoma lecticularia (Hemiptera: Reduviidae) to northwestern Mexico, under laboratory conditions," Acta Tropica, vol. 177, pp. 194-199, 2018.

[10] J. A. Martinez-Ibarra, R. Alejandre-Aguilar, E. ParedesGonzalez et al., "Biology of three species of North American Triatominae (Hemiptera: Reduviidae: Triatominae) fed on rabbits," Memórias do Instituto Oswaldo Cruz, vol. 102, no. 8, pp. 925-930, 2007.

[11] R. Curtis-Robles, L. D. Auckland, K. F. Snowden, G. L. Hamer, and S. A. Hamer, "Analysis of over 1500 triatomine vectors from across the US, predominantly Texas, for Trypanosoma cruzi infection and discrete typing units," Infection, Genetics and Evolution, vol. 58, pp. 171-180, 2018.

[12] K. B. Donnelly, H. C. Lima, and R. G. Titus, "Histologic characterization of experimental cutaneous leishmaniasis in mice infected with Leishmania braziliensis in the presence or absence of sand fly vector salivary gland lysate," The Journal of Parasitology, vol. 84, no. 1, pp. 97-103, 1998.

[13] T. Lestinova, I. Rohousova, M. Sima, C. I. de Oliveira, and P. Volf, "Insights into the sand fly saliva: blood-feeding and immune interactions between sand flies, hosts, and Leishmania," PLoS Neglected Tropical Diseases, vol. 11, no. 7, article e0005600, 2017.

[14] M. T. Mendes, T. M. Carvalho-Costa, M. V. da Silva et al., "Effect of the saliva from different triatomine species on the biology and immunity of TLR-4 ligand and Trypanosoma cruzi-stimulated dendritic cells," Parasites \& Vectors, vol. 9, no. 1, p. 634, 2016.

[15] V. Carregaro, A. Sa-Nunes, T. M. Cunha et al., "Nucleosides from Phlebotomus papatasi salivary gland ameliorate murine collagen-induced arthritis by impairing dendritic cell functions," The Journal of Immunology, vol. 187, no. 8, pp. 4347-4359, 2011.

[16] A. Sá-Nunes, A. Bafica, L. R. Antonelli et al., "The immunomodulatory action of sialostatin $\mathrm{L}$ on dendritic cells reveals its potential to interfere with autoimmunity," The Journal of Immunology, vol. 182, no. 12, pp. 7422-7429, 2009.

[17] H. Horka, V. Staudt, M. Klein et al., "The tick salivary protein sialostatin L inhibits the Th9-derived production of the asthma-promoting cytokine IL-9 and is effective in the 
prevention of experimental asthma," The Journal of Immunology, vol. 188, no. 6, pp. 2669-2676, 2012.

[18] H. Sales-Campos, P. R. de Souza, P. J. Basso et al., "Aedes aegypti salivary gland extract ameliorates experimental inflammatory bowel disease," International Immunopharmacology, vol. 26, no. 1, pp. 13-22, 2015.

[19] H. Lent and P. Wygodzinsky, "Revision of the Triatominae (Hemiptera, Reduviidae), and their significance as vectors of Chagas' disease," Bulletin of the American Museum of Natural History, vol. 163, no. 3, pp. 123-520, 1979.

[20] I. C. Huygen, "Reaction of nitrogen dioxide with Griess type reagents," Analytical Chemistry, vol. 42, no. 3, pp. 407-409, 1970.

[21] B. Coutinho de Sousa, J. Reis Machado, M. V. da Silva et al., "Morinda citrifolia (noni) fruit juice reduces inflammatory cytokines expression and contributes to the maintenance of intestinal mucosal integrity in DSS experimental colitis," Mediators of Inflammation, vol. 2017, Article ID 6567432, 10 pages, 2017.

[22] K. M. Felix, S. Tahsin, and H. J. Wu, "Host-microbiota interplay in mediating immune disorders," Annals of the New York Academy of Sciences, vol. 1417, no. 1, pp. 57-70, 2017.

[23] D. C. Baumgart and S. R. Carding, "Inflammatory bowel disease: cause and immunobiology," The Lancet, vol. 369, no. 9573, pp. 1627-1640, 2007.

[24] R. J. Xavier and D. K. Podolsky, "Unravelling the pathogenesis of inflammatory bowel disease," Nature, vol. 448, no. 7152, pp. 427-434, 2007.

[25] G. Rogler and T. Andus, "Cytokines in inflammatory bowel disease," World Journal of Surgery, vol. 22, no. 4, pp. 382389, 1998.

[26] J. F. Colombel, W. J. Sandborn, P. Rutgeerts et al., “Adalimumab for maintenance of clinical response and remission in patients with Crohn's disease: the CHARM trial," Gastroenterology, vol. 132, no. 1, pp. 52-65, 2007.

[27] S. R. Targan, B. G. Feagan, R. N. Fedorak et al., "Natalizumab for the treatment of active Crohn's disease: results of the ENCORE trial," Gastroenterology, vol. 132, no. 5, pp. 16721683, 2007.

[28] P. J. Basso, M. T. Fonseca, G. Bonfa et al., “Association among genetic predisposition, gut microbiota, and host immune response in the etiopathogenesis of inflammatory bowel disease," Brazilian Journal of Medical and Biological Research, vol. 47, no. 9, pp. 727-737, 2014.

[29] N. S. Ding, A. Hart, and P. De Cruz, "Systematic review: predicting and optimising response to anti-TNF therapy in Crohn's disease - algorithm for practical management," Alimentary Pharmacology \& Therapeutics, vol. 43, no. 1, pp. 30-51, 2016.

[30] M. Allocca, M. Jovani, G. Fiorino, S. Schreiber, and S. Danese, "Anti-IL-6 treatment for inflammatory bowel diseases: next cytokine, next target," Current Drug Targets, vol. 14, no. 12, pp. 1508-1521, 2013.

[31] S. Danese, S. Vermeire, P. Hellstern et al., "Randomised trial and open-label extension study of an anti-interleukin-6 antibody in Crohn's disease (ANDANTE I and II)," Gut, 2017.

[32] H. Ito, M. Takazoe, Y. Fukuda et al., "A pilot randomized trial of a human anti-interleukin-6 receptor monoclonal antibody in active Crohn's disease," Gastroenterology, vol. 126, no. 4, pp. 989-996, 2004.

[33] C. H. Lee, P. Hsu, B. Nanan et al., "Novel de novo mutations of the interleukin-10 receptor gene lead to infantile onset inflammatory bowel disease," Journal of Crohn's \& Colitis, vol. 8, no. 11, pp. 1551-1556, 2014.

[34] J. O. Shim and J. K. Seo, "Very early-onset inflammatory bowel disease (IBD) in infancy is a different disease entity from adultonset IBD; one form of interleukin-10 receptor mutations," Journal of Human Genetics, vol. 59, no. 6, pp. 337-341, 2014.

[35] S. Veenbergen, M. A. van Leeuwen, G. J. Driessen et al., "Development and function of immune cells in an adolescent patient with a deficiency in the interleukin-10 receptor," Journal of Pediatric Gastroenterology and Nutrition, vol. 65, no. 1, pp. e5-e15, 2017.

[36] D. S. Shouval, A. Biswas, J. A. Goettel et al., "Interleukin-10 receptor signaling in innate immune cells regulates mucosal immune tolerance and anti-inflammatory macrophage function," Immunity, vol. 40, no. 5, pp. 706-719, 2014.

[37] N. S. Redhu, V. Bakthavatchalu, E. A. Conaway et al., "Macrophage dysfunction initiates colitis during weaning of infant mice lacking the interleukin-10 receptor," eLife, vol. 6, 2017.

[38] A. Raza, J. W. Crothers, M. M. McGill, G. M. Mawe, C. Teuscher, and D. N. Krementsov, "Anti-inflammatory roles of p38 $\alpha$ MAPK in macrophages are context dependent and require IL-10," Journal of Leukocyte Biology, vol. 102, no. 5, pp. 1219-1227, 2017.

[39] M. Rincon and R. J. Davis, "Regulation of the immune response by stress-activated protein kinases," Immunological Reviews, vol. 228, no. 1, pp. 212-224, 2009.

[40] C. Kim, Y. Sano, K. Todorova et al., "The kinase p38 $\alpha$ serves cell type-specific inflammatory functions in skin injury and coordinates pro- and anti-inflammatory gene expression," Nature Immunology, vol. 9, no. 9, pp. 1019-1027, 2008. 


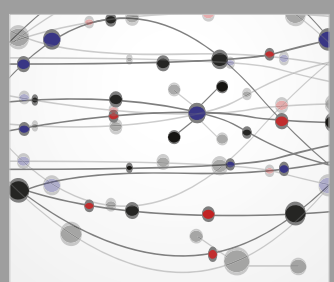

The Scientific World Journal


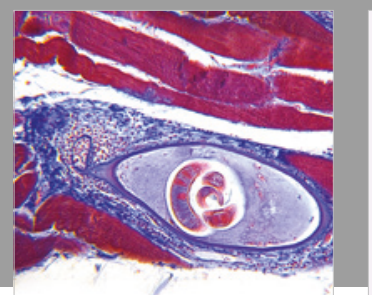

Gastroenterology Research and Practice



\section{Hindawi}

Submit your manuscripts at

www.hindawi.com
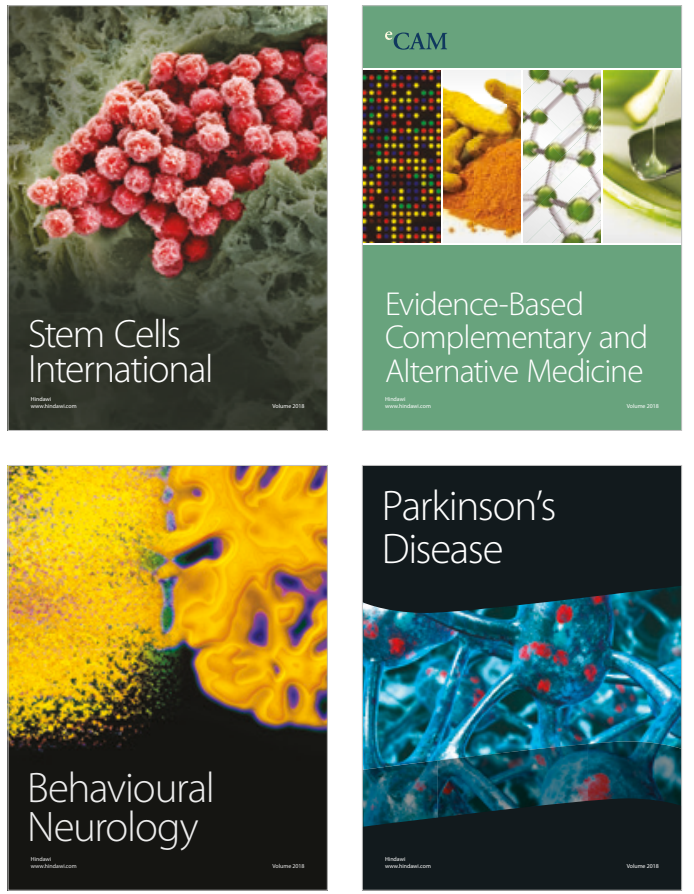

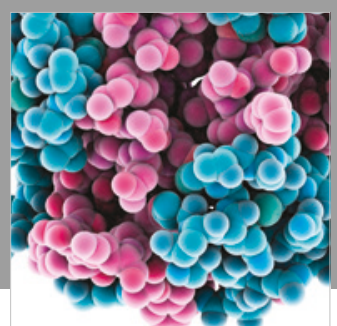

ournal of

Diabetes Research

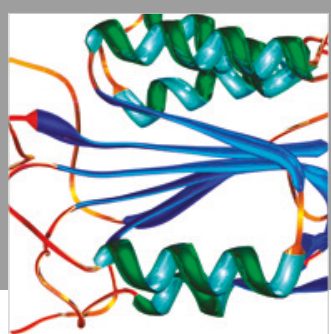

Disease Markers
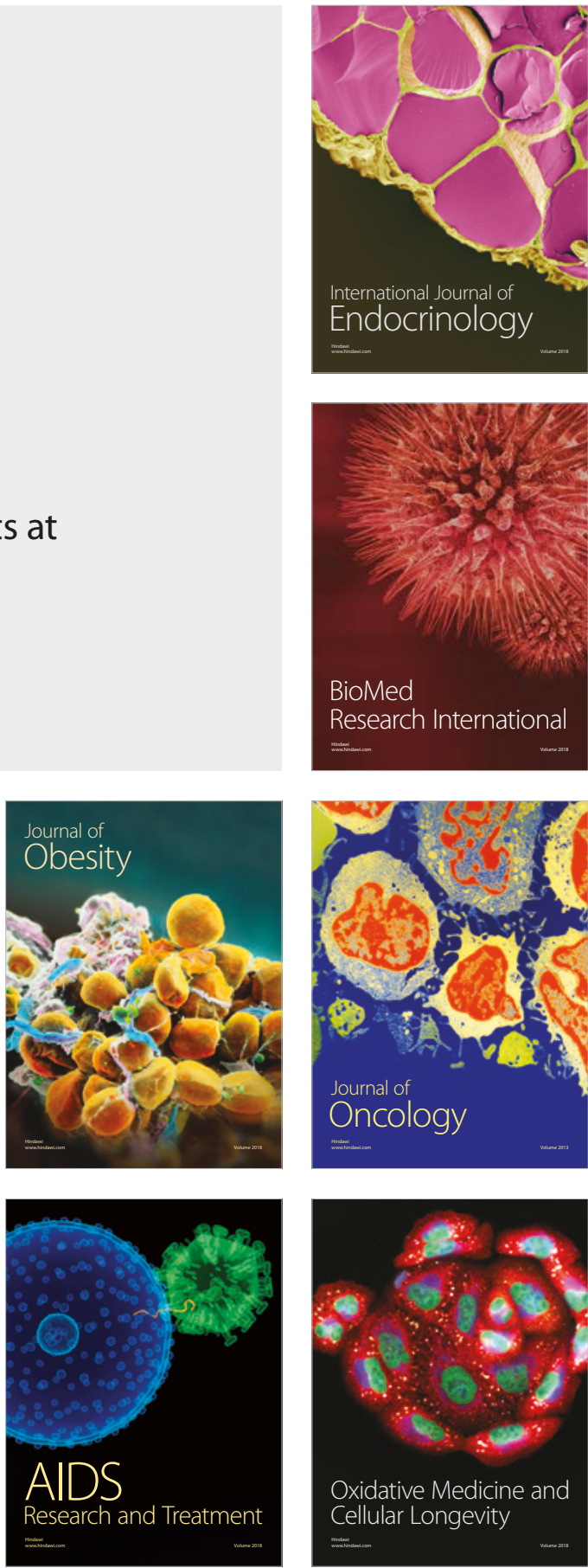